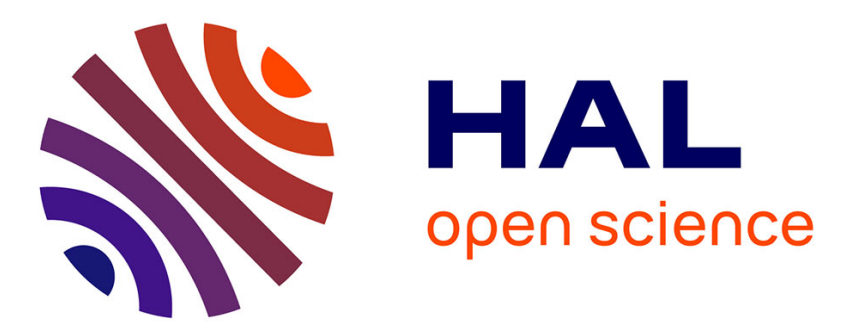

\title{
Whirling frequencies and amplitudes due to deviations of generator shape
}

Niklas L.P. Lundström, J.-O. Jan-Olov Aidanpää

\section{To cite this version:}

Niklas L.P. Lundström, J.-O. Jan-Olov Aidanpää. Whirling frequencies and amplitudes due to deviations of generator shape. International Journal of Non-Linear Mechanics, 2008, 43 (9), pp.933. 10.1016/j.ijnonlinmec.2008.07.002 . hal-00501790

\section{HAL Id: hal-00501790 https://hal.science/hal-00501790}

Submitted on 12 Jul 2010

HAL is a multi-disciplinary open access archive for the deposit and dissemination of scientific research documents, whether they are published or not. The documents may come from teaching and research institutions in France or abroad, or from public or private research centers.
L'archive ouverte pluridisciplinaire HAL, est destinée au dépôt et à la diffusion de documents scientifiques de niveau recherche, publiés ou non, émanant des établissements d'enseignement et de recherche français ou étrangers, des laboratoires publics ou privés. 


\section{Author's Accepted Manuscript}

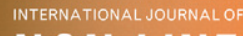

NON-LINEAR

MECHANICS

Whirling frequencies and amplitudes due to deviations of generator shape

Niklas L.P. Lundström, Jan-Olov Aidanpää

PII:

DOI:

S0020-7462(08)00138-8

Reference:

doi:10.1016/j.ijnonlinmec.2008.07.002

NLM 1505

To appear in: International Journal of Non-

Linear Mechanics

Received date: 7 September 2006

Revised date: $\quad 30$ June 2008

Accepted date: 3 July 2008

Cite this article as: Niklas L.P. Lundström and Jan-Olov Aidanpää, Whirling frequencies and amplitudes due to deviations of generator shape, International Journal of Non-Linear Mechanics (2008), doi:10.1016/j.ijnonlinmec.2008.07.002

This is a PDF file of an unedited manuscript that has been accepted for publication. As a service to our customers we are providing this early version of the manuscript. The manuscript will undergo copyediting, typesetting, and review of the resulting galley proof before it is published in its final citable form. Please note that during the production process errors may be discovered which could affect the content, and all legal disclaimers that apply to the journal pertain. 


\title{
WHIRLING FREQUENCIES AND AMPLITUDES DUE TO DEVIATIONS OF GENERATOR SHAPE
}

\author{
Niklas L. P. Lundström* \\ Department of Mathematics and Mathematical Statistics, \\ Umeå University, SE-901 8\%, Sweden \\ Jan-Olov Aidanpää ${ }^{\dagger}$ \\ Division of Computer Aided Design, \\ Department of Applied Physics and Mechanical Engineering, \\ Luleå University of Technology, SE-971 87, Sweden
}

(Dated: June 30, 2008) 


\begin{abstract}
Earlier measurements in large synchronous generators indicate the existence of backward whirling motion, and, also relatively large deviations of shape in both the rotor and the stator. These non-symmetric geometries produce an attraction force between the rotor and the stator, called Unbalanced Magnetic Pull (UMP). The target of this paper is to analyse the whirling frequencies and amplitude of the response for large synchronous generators with a high number of poles, due to deviations of shape in the rotor and stator. A mathematical model is developed to describe the shapes of the rotor and stator, and the corresponding UMP is obtained by using the law of energy conservation. The UMP is analysed due to different deviations of shape. The result gives the average angular frequency and the magnitude of the UMP for certain deviations of shape. From this result, the whirling frequency and the amplitude of the corresponding response can be approximated. Simulations of the response of a Jeffcott rotor model show good agreement with the theoretical results of the UMP for some generator geometries. The conclusion is that different whirling frequencies, both backward and forward whirling, can occur in these machines due to deviations in shape of the generator. Therefore, the shape of the generator can excite resonance vibrations on several other frequencies than the rotation frequency. During maintenance of hydropower generators the shapes of the rotor and stator are frequently measured. The results from this paper can be used to evaluate such measurements and to explain the existence of complicated whirling motion.
\end{abstract}

*Electronic address: niklas.lundstrom@math.umu.se

${ }^{\dagger}$ Electronic address: joa@ltu.se 
Keywords; hydropower, asymmetric, generator, rotor, dynamics

\section{INTRODUCTION}

Hydropower generators have small air-gaps between the rotor and the stator. Usually the gap is about $0.2 \%$ of the stator inner radius. Measurements on the shape of hydropower generators are frequently carried out during maintenance. Measurements done indicate that all hydropower generators are associated with some degree of asymmetry in the air-gap. These asymmetries distort the air-gap flux density distribution and produce an attraction force between the rotor and the stator which is called Unbalanced Magnetic Pull (UMP). The effect of UMP can damage the machine. There are documented cases, e.g. Talas and Toom [1], where the rotor has been in contact with the stator which has been caused by air-gap asymmetries. Measurements also indicate the existence of complicated whirling motions, including both backward and forward whirling, in these machines. A literature survey indicates intensive studies of methods for calculating UMP caused by eccentricity, as well as studies on the vibration characteristics of a rotor system due to UMP. To mention a few, Guo et al. [2] studied the effects of UMP and the vibration level in three-phase generators with any number of pole pairs, Wang et al. [3] studied the free and forced vibrations for rotors of electric motors, Holopainen et al. [4] studied the rotor-dynamic effects of electromechanical interaction on induction motors and Williamson and Abdel-Magied [5] calculated the UMP in induction motors with asymmetrical rotor cages. The latter showed that with an even distribution pattern of bar faults the UMP may be vanishingly small. Tereshonkov [6] determined the angular frequencies of the UMP due to static and dynamic eccentricity, and also due to rotor and stator core ovalities. An overview of the research in the area of modeling and calculating UMP in electrical machines was presented by Frosini and Pennacchi [7]. In [8], Lundström and Aidanpää derived the UMP for an arbitrary disturbed air-gap through the principle of virtual work applied to a simple generator model. They studied the generator shape and proved the existence of stable equilibrium for the rotor for certain cases of deviations of shape. They evaluated the robustness of the generator by simulations of the basin of attraction to attractors without rotor stator contact using a Jeffcott rotor 
model. This paper uses the same simple generator model, and the same derivation of the UMP as in [8]. A power series expansion gives the possibility to mathematically prove a theorem about the angular frequency and the amplitude of the UMP, for certain deviations of shape. Simulations of trajectories using a Jeffcott rotor model extends the result by verifying that attractors behave according to the Theorem even in a number of cases where the mathematical proof does not apply. By comparing to the literature study above, it is reasonable to claim that the results presented here, are new. Since the generator model used is very simple, this paper gives the reader a general and clear guide for understanding the relation between asymmetries in the rotor and complicated whirling motions.

\section{GENERATOR GEOMETRY}

Fig. 1 shows the geometry of the generator model. The generator is treated as a balanced Jeffcott or Laval rotor having a rigid core with length $l_{0}$, mass $\gamma$ and stiffness $k$ of the generator shaft. The rotor rotates counter-clockwise, at a constant angular speed $\omega$. Point $C_{s}$ gives the location of the bearings while point $C_{r}$ is the geometrical center line of the rigid rotor core. The coordinate system has its origin at $C_{s}, r$ is the rotor radius and $s$ is the stator radius. Let $r_{0}$ and $s_{0}$ be the average radius of the rotor and the stator respectively. An arbitrary non-circular shape of the rotor radius, $r$, and the stator radius, $s$, can be described by the Fourier series

$$
\begin{aligned}
& r=r_{0}(z)+\sum_{n=1}^{\infty} \delta_{n}^{r}(z) \cos \left\{n\left(\varphi+\alpha_{n}^{r}(z)\right)\right\} \\
& s=s_{0}(z)+\sum_{m=1}^{\infty} \delta_{m}^{s}(z) \cos \left\{m\left(\varphi+\alpha_{m}^{s}(z)\right)\right\},
\end{aligned}
$$

where

$$
\delta_{n}^{r}(z) \geq 0, \quad \delta_{m}^{s}(z) \geq 0, \quad \sum_{n=1}^{\infty} \delta_{n}^{r}(z)+\sum_{m=1}^{\infty} \delta_{m}^{s}(z)<g_{0}(z)
$$

Here, $g_{0}=s_{0}-r_{0}$ is the average air-gap, $\delta_{n}^{r}$ and $\delta_{m}^{s}$ are referred to as the rotor and stator perturbation parameters, while $\alpha_{n}^{r}$ and $\alpha_{m}^{s}$ are the corresponding phase angles. To simplify notations, it is hereinafter assumed that $\delta_{n}^{r}=\delta_{m}^{s}=0, \forall m, n \in \boldsymbol{N}$, if nothing else is mentioned. $\boldsymbol{N}$ is here the set of all natural numbers. Fig. 2 shows the rotor shape 
for $\delta_{n}^{r}=r_{0} / 3, n=1,2,3,4$, and phase angles $\alpha_{n}^{r}=0$. Note that the stator has the same shape for $\delta_{m}^{s}=\delta_{n}^{r}$ and $m=n$. The cases $\delta_{1}^{r}>0$ and $\delta_{1}^{s}>0$ will correspond to rotor eccentricity and stator eccentricity respectively. Note that, since rotor eccentricity almost surely results in a dynamic eccentricity, it is often referred to as dynamic eccentricity. Similarly, stator eccentricity is often referred to as static eccentricity.

Since dynamic eccentricity is normally small compared to the radius of the air-gap, it is assumed that the perturbed air-gap $(g)$ is

$$
g=s(z, \varphi)-r(z, \varphi)-x \cos \varphi-y \sin \varphi
$$

where $(x, y)$ gives the position of $C_{r}$. Eqs. (1), (2) and (4) give, after adding the $\omega$ rotation,

$$
\begin{aligned}
g & =g_{0}(z)+\sum_{m=1}^{\infty} \delta_{m}^{s}(z) \cos \left\{m\left(\varphi+\alpha_{m}^{s}(z)\right)\right\}-\sum_{n=1}^{\infty} \delta_{n}^{r}(z) \cos \left\{n\left(\varphi+\alpha_{n}^{r}(z)-\omega t\right)\right\} \\
& -x \cos \varphi-y \sin \varphi .
\end{aligned}
$$

Note that when considering the rotor fixed at some $(x, y)$, the case $\delta_{1}^{s}>0$ is not necessary to include. The geometric model is now completed.

\section{UNBALANCED MAGNETIC PULL}

To find an analytical expression for the UMP the two following simplifications are made; the generator will be treated as a continuum and the B-Field (also called magnetic flux density) in the air-gap will be assumed as in [3],

$$
B=\frac{B_{0}(z) g_{0}(z)}{g(x, y, z, t, \varphi)}
$$

where $B_{0}$ is the uniformly distributed B-field for a perfect circular geometry, i.e. $g=g_{0}$. These assumptions means that the effect of the poles are not taken into account and are justified since there is a large number of poles in a hydropower generator. Based on the theory of magnetic fields [9], the potential energy stored in the air-gap can be expressed as

$$
E=\int_{\text {air-gap }} \frac{B(x, y, z, t, \varphi)^{2}}{2 \mu_{0}} d V
$$

where $\mu_{0}$ is the permeability of air. Next, consider a volume element $d V$ as shown in Fig. 3. According to Eqs. (7) and (6), the potential energy $(\delta E)$ reserved in $d V$ is given by

$$
\delta E=\frac{B_{0}(z)^{2} g_{0}(z)^{2}}{2 \mu_{0} g(x, y, z, t, \varphi)^{2}} d V .
$$


Eq. (8) shows that if the air-gap is disturbed from the current value $g$ to a new value $g+d g$, $\delta E$ will increase if $d g<0$ and decrease if $d g>0$. Let $d E_{\text {mech }}$ be increments of mechanical energy input to $d V$ and $d E_{\text {electric }}$ the electric energy output from $d V$. When considering the energy conversion between the magnetic and mechanical fields over an infinitesimal period of time, the law of energy conservation requires, after neglecting losses

$$
d E_{\text {mech }}=d(\delta E)+d E_{\text {electric }} .
$$

As in the case of eccentricity [3], it is assumed that the electric energy output from the air-gap is independent of the air-gap variations, thus $d E_{\text {electric }}=0$. If $d g<0$, then $d(\delta E)=d E_{\text {mech }}>0$. Since the mechanical energy input increases when $g$ decreases, a force acting in the radial direction has to be present. Denote this force by $d f$. Then, the virtual work done by this force is $d f d g=-d(\delta E)$, which gives

$$
d f=-\frac{d}{d g}(\delta E) .
$$

In Eq. (8), note that, since $d V=r d r d z d \varphi$, the potential energy $\delta E$ will increase if $r$ increases when $g$ is constant. This small change in $d f$ cannot be considered in Eq. (10). But, since the change of $g$ and $r$ is of approximately the same size and $g<<r$, the change of $\delta E$ due to $r$ is negligible, and therefore, to simplify the calculations it is assumed that $d V=u_{0} d r d z d \varphi$, where $u_{0}=\left(r_{0}+s_{0}\right) / 2$, and $d r=g$. Eq. (8) then yields

$$
\delta E=\frac{B_{0}(z)^{2} g_{0}(z)^{2} u_{0}(z)}{2 \mu_{0} g(x, y, z, t, \varphi)} d z d \varphi
$$

According to Eq. (10), the force $d f$ is given by

$$
d f=-\frac{d}{d g}(\delta E)=\frac{B_{0}(z)^{2} g_{0}(z)^{2} u_{0}(z)}{2 \mu_{0} g(x, y, z, t, \varphi)^{2}} d z d \varphi .
$$

Hence, the total forces in the $x$ - and $y$-direction can be expressed as

$$
\begin{aligned}
& f_{x}=\frac{1}{2 \mu_{0}} \int_{0}^{2 \pi} \int_{0}^{l_{0}} \frac{B_{0}(z)^{2} g_{0}(z)^{2} u_{0}(z)}{g(x, y, z, t, \varphi)^{2}} \cos \{\varphi\} d z d \varphi, \\
& f_{y}=\frac{1}{2 \mu_{0}} \int_{0}^{2 \pi} \int_{0}^{l_{0}} \frac{B_{0}(z)^{2} g_{0}(z)^{2} u_{0}(z)}{g(x, y, z, t, \varphi)^{2}} \sin \{\varphi\} d z d \varphi .
\end{aligned}
$$

The generator geometry and the B-field are from now on assumed constant in $z$ through the generator length $l_{0}$. This is the case through the rest of the paper. For ideal circular 
generator geometry and $y=0$, the integral in Eq. (13) can be solved analytically to yield

$$
f_{x}=\frac{l_{0} g_{0}^{2} B_{0}^{2} u_{0}}{2 \mu_{0}} \int_{0}^{2 \pi} \frac{\cos \varphi}{\left(g_{0}-x \cos \varphi\right)^{2}} d \varphi=k_{m} \frac{x}{\left(1-\frac{x^{2}}{g_{0}^{2}}\right)^{\frac{3}{2}}} .
$$

Here, the magnetic stiffness $\left(k_{m}\right)$, is defined as

$$
k_{m}=\frac{\pi l_{0} B_{0}^{2} u_{0}}{\mu_{0} g_{0}}
$$

Eq. (15) is similar to results obtained by Wang et al. [3] and Sadarangani [10].

\section{EQUATIONS OF MOTION}

The equations of motion for the forced Jeffcott rotor is non-autonomous and nonlinear and consists of two second order differential equations

$$
\begin{aligned}
\gamma \ddot{x}+c \dot{x}+k x & =f_{x}(x, y, t), \\
\gamma \ddot{y}+c \dot{y}+k y & =f_{y}(x, y, t) .
\end{aligned}
$$

Here, $\gamma$ is the mass of the rotor, $k$ is the stiffness of the rotor shaft and $c$ being a linear viscous damping.

In non-dimensional form, system (17) yields

$$
\begin{aligned}
X^{\prime \prime}+2 \zeta X^{\prime}+X & =F_{X}(X, Y, \tau) \\
Y^{\prime \prime}+2 \zeta Y^{\prime}+Y & =F_{Y}(X, Y, \tau)
\end{aligned}
$$

Here, the non-dimensional quantities

$$
\begin{gathered}
X=\frac{x}{g_{0}}, \quad Y=\frac{y}{g_{0}}, \quad \Delta_{n}^{R}=\frac{\delta_{n}^{r}}{g_{0}}, \quad \Delta_{m}^{S}=\frac{\delta_{m}^{s}}{g_{0}} \\
\zeta=\frac{c}{2 \sqrt{k \gamma}}, \quad G=\frac{g(x, y, t, \varphi)}{g_{0}}, \quad \Omega=\omega \sqrt{\frac{\gamma}{k}}, \quad \tau=t \sqrt{\frac{k}{\gamma}},
\end{gathered}
$$

have been introduced and $\tau$ is a non-dimensional time. The air-gap $G$, and the forces $F_{X}$ and $F_{Y}$ yield

$$
F_{X}=\frac{k_{m}}{2 \pi k} \int_{0}^{2 \pi} \frac{\cos \varphi}{G(X, Y, \tau, \varphi)^{2}} d \varphi
$$




$$
\begin{gathered}
F_{Y}=\frac{k_{m}}{2 \pi k} \int_{0}^{2 \pi} \frac{\sin \varphi}{G(X, Y, \tau, \varphi)^{2}} d \varphi \\
G=1+\sum_{m=1}^{\infty} \Delta_{m}^{S} \cos \left\{m\left(\varphi+\alpha_{m}^{s}\right)\right\}-\sum_{n=1}^{\infty} \Delta_{n}^{R} \cos \left\{n\left(\varphi+\alpha_{n}^{r}-\Omega \tau\right)\right\} \\
-X \cos \varphi-Y \sin \varphi .
\end{gathered}
$$

\section{PROPERTIES OF THE UMP}

To gain understanding of the responses from the UMP due to different deviations of shape, properties of the UMP will be investigated in this section.

\section{A. SIMULATIONS}

The angular frequency of the UMP is given by

$$
\Theta=\frac{F_{Y}^{\prime} F_{X}-F_{X}^{\prime} F_{Y}}{F_{X}^{2}+F_{Y}^{2}} .
$$

Here, the prime represents differentiation with respect to time $\tau, F_{X}$ and $F_{Y}$ are given by Eqs. (21) and (22). The MATLAB function QUAD was used to evaluate the integrals. To get an introduction to MATLAB, see for example Gilat [11]. The numerical values used are from an 18 MW hydropower generator and are given in Table I.

To visualize the influence of shape perturbations on the amplitude, six different shape perturbations are shown in Fig. 4 for a concentric rotor $(X=Y=0)$. Fig. 5(a) shows the result from a simulation of the largest value of the UMP during one revolution of the rotor when $X=Y=0$, i.e.

$$
\max _{\Omega \tau \in[0,2 \pi]}\left\{\sqrt{F_{X}^{2}+F_{Y}^{2}}\right\} .
$$

Fig. 5(b) shows the generator geometry for the case $\Delta_{10}^{S}>0, \Delta_{9}^{R}>0$. The Figure shows that if $m-n= \pm 1$, several minima of the air-gap will occur near the absolute minimum, and therefore, larger amplitudes of the UMP will occur in these cases. This can be compared with the result presented in Fig 5(a). The same argument can explain the larger amplitudes on the lines $2 m-n= \pm 1$ and $m-2 n= \pm 1$, which also can be observed in Fig 5(a). These phenomena will be analytically proved in the next section. By studying Fig. 5(b) the 
existence of $m n$-combinations giving no UMP can be realized, e.g. the cases $m=n$. All such combinations were derived by Lundström and Aidanpää in [8].

\section{B. ANALYSIS}

In this section, a theorem describing how the average angular frequency and amplitude of the UMP are depending on different deviations of shape in the generator will be proved. Consider the air-gap given by Eq. (23) including the $N$ first rotor perturbation parameters $\Delta_{n}^{R}$ and the $M$ first stator perturbation parameters $\Delta_{m}^{S}$, i.e.

$$
\begin{aligned}
G & =1+\sum_{m=1}^{M} \Delta_{m}^{S} \cos \left\{m\left(\varphi+\alpha_{m}^{s}\right)\right\}-\sum_{n=1}^{N} \Delta_{n}^{R} \cos \left\{n\left(\varphi+\alpha_{n}^{r}-\Omega \tau\right)\right\} \\
& -X \cos \varphi-Y \sin \varphi .
\end{aligned}
$$

The forces $F_{X}$ and $F_{Y}$ given by Eqs. (21) and (22) can be analysed using the Maclaurin series

$$
\frac{1}{(1-\epsilon)^{2}}=1+2 \epsilon+3 \epsilon^{2}+\ldots+(q+1) \epsilon^{q}+\ldots
$$

with

$$
\begin{aligned}
\epsilon & =-\sum_{m=1}^{M} \Delta_{m}^{S} \cos \left\{m\left(\varphi+\alpha_{m}^{s}\right)\right\}+\sum_{n=1}^{N} \Delta_{n}^{R} \cos \left\{n\left(\varphi+\alpha_{n}^{r}-\Omega \tau\right)\right\} \\
& +X \cos \varphi+Y \sin \varphi
\end{aligned}
$$

From Eqs. (22) and (27), $F_{Y}$ can be expressed as

$$
\begin{aligned}
F_{Y} & =\frac{k_{m}}{2 \pi k} \int_{0}^{2 \pi}\left(1+2 \epsilon+3 \epsilon^{2}+\ldots+(q+1) \epsilon^{q}+\ldots\right) \sin \{\varphi\} d \varphi \\
& =F_{Y}^{1}+F_{Y}^{2}+\ldots+F_{Y}^{q}+\ldots
\end{aligned}
$$

The analysis is now continued by approximating $F_{Y}^{q}, q \in \boldsymbol{N}$. From Eqs. (28), (29) and the multinomial theorem, $F_{Y}^{q}$ yields, with $\kappa=k_{m}(q+1) /(2 \pi k)$,

$$
\begin{aligned}
F_{Y}^{q} & =\kappa \int_{0}^{2 \pi} \epsilon^{q} \sin \{\varphi\} d \varphi \\
& =\kappa \int_{0}^{2 \pi}\left\{\sum_{j_{1}+\cdots+j_{A}=q} \frac{q !}{j_{1} ! \ldots j_{A} !} a_{1}^{j_{1}} \ldots a_{A}^{j_{A}}\right\} \sin \{\varphi\} d \varphi,
\end{aligned}
$$


where $A=N+M+2$ are the total number of terms in $\epsilon$ and

$$
\begin{aligned}
a_{n} & =\Delta_{n}^{R} \cos \left\{n\left(\varphi+\alpha_{n}^{r}-\Omega \tau\right)\right\}, \quad n=1 \ldots N, \\
a_{N+m} & =-\Delta_{m}^{S} \cos \left\{m\left(\varphi+\alpha_{m}^{s}\right)\right\}, \quad m=1 \ldots M, \\
a_{A-1} & =X \cos \varphi \\
a_{A} & =Y \sin \varphi .
\end{aligned}
$$

To proceed with the analysis, it is assumed that

$$
X=Y=\Delta_{m}^{S}=\Delta_{n}^{R}=0, \quad \text { for all } \quad m \in \boldsymbol{N} \backslash m^{\prime}, n \in N \backslash n^{\prime}
$$

This means that all except one rotor perturbation parameter $\left(\Delta_{n^{\prime}}^{R}\right)$, one stator perturbation parameter $\left(\Delta_{m^{\prime}}^{S}\right)$ together with the lateral displacement of the rotor, are set to zero. This assumption is more relevant the better the inequality

$$
|X|+|Y|+\sum_{m \in N \backslash m^{\prime}} \Delta_{m}^{S}+\sum_{n \in N \backslash n^{\prime}} \Delta_{n}^{R} \ll<\left(\Delta_{n^{\prime}}^{R}\right)^{q},\left(\Delta_{m^{\prime}}^{S}\right)^{q} .
$$

is satisfied for the machine in question. Note that for every realistic machine (Not perfect in the sense that the left hand side in inequality (33) is non-zero), inequality (33) also gives an upper bound of $q$. (I.e. the order of terms in Eq. (27) to be included in the analysis.) From now on the prime is dropped to simplify the notation. Thus $m^{\prime}=m$ and $n^{\prime}=n$. Moreover, let $\Delta_{n^{\prime}}^{R}=\Delta_{R}$ and $\Delta_{m^{\prime}}^{S}=\Delta_{S}$. Since both the rotor and stator are perturbed by only one perturbation parameter each, and the rotor rotates with a constant angular speed, the phase angles can be chosen arbitrary. Therefore, to exclude an alternating sign, the phase angles are set to $\alpha_{m}^{s}=\pi / m$ and $\alpha_{n}^{r}=0$. Then Eq. (30) can be simplified to

$$
\begin{aligned}
F_{Y}^{q} & =\kappa \int_{0}^{2 \pi}\left\{\sum_{j=0}^{q}\left(\begin{array}{l}
q \\
j
\end{array}\right)\left(\Delta_{R} \cos \{n(\varphi-\Omega \tau)\}\right)^{j}\left(\Delta_{S} \cos \{m \varphi\}\right)^{q-j}\right\} \sin \{\varphi\} d \varphi \\
& =\kappa\left\{\sum_{j=0}^{q}\left(\begin{array}{l}
q \\
j
\end{array}\right) \Delta_{R}^{j} \Delta_{S}^{q-j} \int_{0}^{2 \pi} \cos ^{j}\{n(\varphi-\Omega \tau)\} \cos ^{q-j}\{m \varphi\} \sin \{\varphi\} d \varphi\right\} .
\end{aligned}
$$

Consider the trigonometric identities

$$
\cos ^{2 i} \alpha=\left(\begin{array}{c}
2 i \\
i
\end{array}\right) \frac{1}{2^{2 i}}+\frac{1}{2^{2 i-1}} \sum_{p=1}^{i}\left(\begin{array}{c}
2 i \\
i-p
\end{array}\right) \cos \{2 p \alpha\},
$$




$$
\cos ^{2 i-1} \alpha=\frac{1}{2^{2 i-2}} \sum_{p=1}^{i}\left(\begin{array}{c}
2 i-1 \\
i-p
\end{array}\right) \cos \{(2 p-1) \alpha\} .
$$

By using Eqs.(35) and (36), the integrals in Eq. (34) can be simplified in the following way. Assume that both $j$ and $q-j$ are even, then by Eq. (35) and symmetries of the trigonometric functions,

$$
\begin{aligned}
& \int_{0}^{2 \pi} \cos ^{j}\{n(\varphi-\Omega \tau)\} \cos ^{q-j}\{m \varphi\} \sin \{\varphi\} d \varphi \\
= & \frac{1}{2^{q-2}} \int_{0}^{2 \pi} \sum_{p=1}^{\frac{j}{2}}\left(\begin{array}{c}
j \\
\frac{j}{2}-p
\end{array}\right) \cos \{2 p n(\varphi-\Omega \tau)\} \sum_{p=1}^{\frac{q-j}{2}}\left(\begin{array}{c}
q-j \\
\frac{q-j}{2}-p
\end{array}\right) \cos \{2 p m \varphi\} \sin \{\varphi\} d \varphi \\
= & \frac{1}{2^{q-2}} \int_{0}^{2 \pi} \sum_{s_{1} \in S_{1}}\left(\begin{array}{c}
j \\
\frac{j-s_{1}}{2}
\end{array}\right) \cos \left\{s_{1} n(\varphi-\Omega \tau)\right\} \sum_{s_{2} \in S_{2}}\left(\begin{array}{c}
q-j \\
\frac{q-j-s_{2}}{2}
\end{array}\right) \cos \left\{s_{2} m \varphi\right\} \sin \{\varphi\} d \varphi,
\end{aligned}
$$

where the substitution $s_{1}=2 p$ is made in the first sum, $s_{2}=2 p$ in the second sum and $S_{1}$ is the set of even integers from 2 to $j$ while $S_{2}$ is the set of even integers from 2 to $q-j$. By similar calculations it follows from Eq. (36) that Eq. (37) is true for all $q$ and $j \leq q$, with odd integers from 1 to $j$ in the set $S_{1}$ if $j$ is odd, and similarly odd integers in the set $S_{2}$ if $q-j$ is odd. $F_{Y}^{q}$ can now be expressed as

$$
\begin{array}{r}
F_{Y}^{q}=\frac{\kappa}{2^{q-2}}\left\{\sum_{j=0}^{q}\left(\begin{array}{c}
q \\
j
\end{array}\right) \Delta_{R}^{j} \Delta_{S}^{q-j} \int_{0}^{2 \pi} \sum_{s_{1} \in S_{1}}\left(\begin{array}{c}
j \\
\frac{j-s_{1}}{2}
\end{array}\right) \cos \left\{s_{1} n(\varphi-\Omega \tau)\right\}\right. \\
\left.\sum_{s_{2} \in S_{2}}\left(\begin{array}{c}
q-j \\
\frac{q-j-s_{2}}{2}
\end{array}\right) \cos \left\{s_{2} m \varphi\right\} \sin \{\varphi\} d \varphi\right\} .
\end{array}
$$

From Eq. (38) it can be seen that the following is true;

(i) The terms in the integral corresponding to $s_{1}$ and $s_{2}$ can be found in $F_{Y}^{q-2}$ if $s_{1}$ and $s_{2}$ satisfies $j-s_{1}+(q-j)-s_{2}=2$.

(ii) If $\Delta_{R}$ and $\Delta_{S}$ are small enough, the terms in $F_{Y}^{q-2}$ will dominate the corresponding terms in $F_{Y}^{q}$ since then $\Delta_{R}^{i}>>\Delta_{R}^{\hat{i}}$ if $\hat{i}>i$ and the same is true for $\Delta_{S}$.

(iii) The reasoning in $(i)$ holds with $j-s_{1}+q-j-s_{2}=2$ replaced by $j-s_{1}+q-j-s_{2}=p$ and $F_{Y}^{q-2}$ replaced by $F_{Y}^{q-p}$ when $p=2,4,6, \ldots$ and $p<q$. 
From $(i)-($ iii $)$ above it is concluded that all terms in the integral in Eq. (38) except the terms corresponding to $s_{1}=j$ and $s_{2}=q-j$ can be neglected. Hence,

$$
F_{Y}^{q} \approx \frac{\kappa}{2^{q-2}}\left\{\sum_{j=0}^{q}\left(\begin{array}{l}
q \\
j
\end{array}\right) \Delta_{R}^{j} \Delta_{S}^{q-j} \int_{0}^{2 \pi} \cos \{j n(\varphi-\Omega \tau)\} \cos \{(q-j) m \varphi\} \sin \{\varphi\} d \varphi .\right\}
$$

The integral in (39) can be simplified further. Properties of the trigonometric functions give

$$
\begin{aligned}
& \int_{0}^{2 \pi} \cos \{j n(\varphi-\Omega \tau)\} \cos \{(q-j) m \varphi\} \sin \{\varphi\} d \varphi \\
= & \sin \{j n \Omega \tau\} \int_{0}^{2 \pi} \sin \{j n \varphi\} \cos \{(q-j) m \varphi\} \sin \{\varphi\} d \varphi \\
= & \sin \{j n \Omega \tau\} \int_{0}^{2 \pi} \frac{1}{4}(\cos \{(j n-(q-j) m-1) \varphi\}-\cos \{(j n-(q-j) m+1) \varphi\} \\
& \quad+\cos \{(j n+(q-j) m-1) \varphi\}-\cos \{(j n+(q-j) m+1) \varphi\}) d \varphi \\
= & \sin \{j n \Omega \tau\} \frac{\pi \Gamma^{q, j}}{2} .
\end{aligned}
$$

In Eq. (40), $\Gamma^{q, j}$ is defined for $1 \leq q, 0 \leq j \leq q$ according to

$$
\Gamma^{1, j}=\left\{\begin{array}{l}
2, \text { if } \max (m, n)=1 \\
0, \text { otherwise }
\end{array}\right.
$$

and for $q \geq 2$,

$$
\Gamma^{q, j}= \begin{cases}\mp 1, & \text { if }(q-j) m-j n= \pm 1 \\ 0, & \text { otherwise. }\end{cases}
$$

Recall that $\kappa=k_{m}(q+1) /(2 \pi k)$. From Eqs. (39) and (40) $F_{Y}^{q}$ can be written as

$$
F_{Y}^{q} \approx \frac{(q+1) k_{m}}{2^{q} k} \sum_{j=0}^{q}\left(\begin{array}{l}
q \\
j
\end{array}\right) \Delta_{R}^{j} \Delta_{S}^{q-j} \sin \{j n \Omega \tau\} \Gamma^{q, j},
$$

where $\Gamma^{q, j}$ is given by Eqs. (41) and (42). Applying the same procedure for $F_{X}$ yields

$$
F_{X}^{q} \approx \frac{(q+1) k_{m}}{2^{q} k} \sum_{j=0}^{q}\left(\begin{array}{c}
q \\
j
\end{array}\right) \Delta_{R}^{j} \Delta_{S}^{q-j} \cos \{j n \Omega \tau\}\left|\Gamma^{q, j}\right| .
$$

Let $\widehat{\Theta}$ be the average value of the angular frequency of the UMP. Define

$$
\psi=\frac{\widehat{\Theta}}{\Omega},
$$

then Eqs. (43) and (44) prove the following theorem about the UMP. 
Theorem V.1 Consider a generator for which the perturbations of shape are small enough and for which inequality (33) is satisfied for $q=1,2, \ldots, q^{\prime}$. Then assumption (32) is reasonable and it follows that for deviations of shape with mn-combinations on the lines

$$
(q-j) m-j n= \pm 1
$$

where $q=1,2, \ldots, q^{\prime}$ and $j=0,1, \ldots, q$, the UMP has the following properties;

(i) The average angular frequency satisfies $\psi=\mp j n$.

(ii) The amplitude will be proportional to $\Delta_{R}^{j} \Delta_{S}^{q-j}$.

(iii) For mn-combinations outside the lines for all q, the UMP is zero.

The last statement was also shown by Lundström and Aidanpää in [8]. They showed that $m n$-combinations outside the lines for all $q$ are the points in the $m n$-plane where it is possible to find integers $p_{n}$ and $p_{m}$ such that

$$
p=\frac{n}{p_{n}}=\frac{m}{p_{m}} \geq 2, \quad p \text { integer. }
$$

To clarify the results from Theorem V.1, the average angular frequency divided by the spinning frequency $(\psi)$ is illustrated in Table II for $m, n \in[1,10]$.

The amplitude is illustrated for $m, n \in[1,10]$ including up to fifth order terms $(q \leq 5)$ in Fig. 6. This can be compared with the simulated results shown in Fig. 5(a). Note from Theorem V.1 that the amplitude is largest for $q=1$, and decreases for increasing $q$.

\section{SIMULATIONS OF THE RESPONSE}

In this section simulations of trajectories to system (18) are carried out using a forth order Runge-Kutta method. The force integrals given by Eqs. (21) and (22) are solved numerically at each time step by Simpson's rule using 200 intervals. To avoid transients, over 100 periods of the UMP (period according to Theorem V.1) are simulated before plotting. The initial condition is $X=Y=X^{\prime}=Y^{\prime}=0$ through all simulations.Fig. 7 shows the periodic attractors for 60 cases of shape perturbations. Four different magnitudes of shape perturbation are considered for each of the $15 m n$-combinations. Since eccentricities $(m=1$ 
or $n=1$ ) give a considerably larger UMP than other cases, smaller perturbations are chosen for the eccentricities. By assuming

$$
f_{x}(x, y, t)=k_{m} x+h_{x}, \quad f_{y}(x, y, t)=k_{m} y+h_{y}
$$

where $h_{x}$ and $h_{y}$ are arbitrary functions independent of $x$ and $y$, system (17) becomes linear. This assumption is relevant since Eq. (15) has an almost linear behaviour in $x$ for $x<0.1 g_{0}$. By using the linear UMP given by Eq. (47) the damped natural frequency is calculated according to

$$
\omega_{d}=\sqrt{\frac{k-k_{m}}{\gamma}-\left(\frac{c}{2 \gamma}\right)^{2}} \approx 44.6 \mathrm{rad} / \mathrm{s} .
$$

The numerical values of $k, k_{m}$ and $\gamma$ can be found in Table I. The damping $c$ is chosen to give the damping ratio $\zeta=0.1$.

Fig. 8 shows the largest amplitude of the response of the rotor $\left(\max \left\{\sqrt{X^{2}+Y^{2}}\right\}\right)$ as a function of spin frequency $\omega$ for four different $m n$-combinations at the same amount of perturbation.

\section{DISCUSSION}

In Section III, the UMP is derived according to the principle of virtual work. The generator is treated as a continuum and the B-field is assumed according to Eq. 6. These are strong simplifications, but are justified since there is a large number of poles in a hydropower generator (the generator considered in this paper has 44 poles). Even if this way of finding the UMP is a strong simplification, it is justified since more detailed models including arbitrary disturbed air-gaps and giving analytically the UMP are not available today. Due to this simple generator model, this work gives a guide for understanding the relation between asymmetries in the rotor and complicated whirling motions, rather than an attempt to imitate the behaviour of a special machine. Fig 4 in Section VA shows that the $m n$-combination $[m, n]=[1,1]$ gives an alternating angular velocity $\Theta$ of the UMP for the case $\Delta_{1}^{S}=0.1, \Delta_{1}^{R}=0.05$. When $[m, n]=[1,1]$, this occurs if $\Delta_{1}^{S}>\Delta_{1}^{R}$. This is shown in Theorem V.1 when considering $F_{X}^{1}$ and $F_{Y}^{1}$. The response in this case will be a forward whirling orbit, having a periodicity equal to the spinning frequency, independent on whether $\Theta$ is alternating or not. Fig. 7 in Section VI illustrates periodic attractors 
for 60 cases of shape perturbations. In all cases, a comparison to Theorem V.1 gives a satisfactory result. To mention a few;

(i) All whirling frequencies satisfy Table II.

(ii) Attractors corresponding to $m=1$ and $n=1$ have a large amplitude, see Figs. 7 (a), (b), (c), (d), (g), (j), (m).

(iii) The amplitudes are larger for attractors to geometries having an $m n$-combination on the lines $m-n= \pm 1$, i.e. in Figs. 7 (e), (h), (i), (k), (l), than amplitudes to attractors in Figs. 7 (f), (n), (o).

(iv) In Figs. 7 (e), (h), (i), (k), (l) the amplitudes are nearly independent of whether $\Delta_{n}^{S}$ or $\Delta_{n}^{R}$ are larger, but in Fig. 7 (f), the case of larger $\Delta_{2}^{S}$ gives the largest amplitude, and in Figs. 7 (n), (o), the case of a larger $\Delta_{n}^{R}$ gives the largest amplitudes.

These simulations show that attractors behave according to the properties of the UMP proved in Theorem V.1, and also point out that the results from the theorem are valid for some cases of deviations in shape even if inequality (33) is not satisfied.

From Fig. 8, Section VI, it is shown that resonance occurs at different spinning frequencies due to the shape perturbation considered. The reason is that for a certain $m n$-combination of perturbation, the UMP has a corresponding angular frequency according to Theorem V.1. Compare the result from the theorem to Fig. 8 and note from Eq. (48) that the damped natural frequency, $\omega_{d} \approx 44.6 \mathrm{rad} / \mathrm{s}$. Observe that the curve corresponding to $[m, n]=[3,2]$ has two peaks.

The larger peak is explained in Theorem V.1 by considering second order terms $(q=2)$, while the smaller peak is explained by considering third order terms $(q=3)$. See Fig. 5(a). Note that lines corresponding to $q=2$ intersect with lines corresponding to $q=3$ at the points $[m, n]=[3,2]$ and $[2,3]$. At $[m, n]=[3,2], q=2$ gives $\Psi=-2$, while $q=3$ gives $\Psi=4$, hence, two peaks can occur, approximately at $\omega=44.6 / 2$, and $\omega=44.6 / 4$. At $[m, n]=[2,3], q=2$ gives $\Psi=3$, while $q=3$ gives $\Psi=-3$, thus, one peak occurs at approximately $\omega=44.6$ / 3.In this paper, deviations of shape are analysed of which one 
rotor perturbation parameter and one stator perturbation parameter are dominating. Other perturbation parameters together with $X$ and $Y$ are assumed to be sufficiently small in order to be neglected. Otherwise the problem will be to complicated and a general approach is difficult.

\section{CONCLUSIONS}

Earlier measurements on large synchronous generators have indicated the existence of nonsymmetric geometries, which produce an attraction force between the rotor and the stator, called Unbalanced Magnetic Pull (UMP). These non-symmetric geometries consists of relatively large deviations of shape in both rotor and stator. In this paper, the amplitude and the average angular frequency of the UMP due to generator shape are found analytically for certain cases of deviations of shape. The results are proved mathematically and are presented in Theorem V.1. These results explain that different whirling frequencies, backward as well as forward whirling, can occur in large synchronous generators due to deviations in the generator shape. Simulations of trajectories using a Jeffcott rotor model show good agreement between the whirling, amplitude of the response and the properties of the UMP given in Theorem V.1. The resulting periodic solutions can be complicated for some geometries. See Fig. 7 (h). In addition the simulations extends the result by pointing out that attractors behave according to Theorem V.1 in a number of cases although the mathematical proof does not apply. The results in this paper clarifies which whirling motion and amplitude can be expected in a machine with a given deviation of shape. Since the angular frequency of the UMP is dependent of the deviations in shape, different spin frequencies can give resonance. See Fig. 8. Since UMP can cause large vibrations in hydropower generators which in turn can cause damage to the machine, the shape of the rotor and stator is frequently measured during maintenance. The results from this paper can be used to evaluate such measurements and also provide guidance on which tolerances that are of greater importance than others, when constructing new machines. 


\section{ACKNOWLEDGEMENT}

Elforsk AB and the Swedish Energy Authority "by the Elektra research program" are acknowledged for the financial support of this project.

[1] P. Talas and P. Toom, Dynamic Measurement and Analysis of Air-Gap Variations in Large Hydroelectric Generators. IEEE Transactions on Power Apparatus and Systems. 9, 102 (1983).

[2] D. Guo, F. Chu and D.Chen, The Unbalanced Magnetic Pull and its Effects on Vibration in a Three-Phase Generator With Eccentric Rotor. Journal of Sound and Vibration. 2, 254 (2002).

[3] Y. Wang, G. Sun and L. Huang, Magnetic Field-induced Nonlinear Vibration of an Unbalanced Rotor. Proceedings of ASME Design Engineering Division. 2 (2003).

[4] T. P. Holopainen, A. Tenhunen and A. Arkkio, Electromechanical Interaction in Rotordynamics of Cage Induction Motors. Journal of Sound and Vibration. 3 - 5, 284 (2005).

[5] S. Williamson and M. A. S. Abdel-Magied, Unbalanced Magnetic Pull in Induction Motors With Asymmetrical Rotor Cages. IEE Conference Publication. 254 (1985).

[6] V. A. Tereshonkov, Magnetic Forces in Electric Machines with Air Gap Eccentricities and Core Ovalities. Elektrotekhnika. 9, 60 (1989).

[7] L. Frosini and P. Pennacchi, Detection and Modelling of Rotor Eccentricity in Electric Machines - an Overview. IMechE Event Publications. 2 (2004).

[8] N. L. P. Lundström and J. -O. Aidanpää, Dynamic Consequences of Electromagnetic Pull due to Deviations in Generator Shape, Journal of Sound and Vibration. 1 - 2, 301 (2007).

[9] R. K. Wangsness, Electromagnetic Fields, 2nd edition, Hamilton Printing Company, United States (1986).

[10] C. Sadarangani, Electrical Machines Design and Analysis of Induction and Permanent Magnet Motors. Royal Institute of Technology, Stockholm (2000).

[11] A. Gilat, MATLAB: An Introduction with Applications. Hoboken, NJ: Wiley, cop. (2005). 
TABLE I: Numerical values from the $18 \mathrm{MW}$ hydropower generator.

\begin{tabular}{lll}
\hline \hline$s_{0}$ & Average stator inner radius & $2.775 \mathrm{~m}$ \\
$l_{0}$ & Length of the rotor core & $1.18 \mathrm{~m}$ \\
$g_{0}$ & Average radial air-gap length & $0.0125 \mathrm{~m}$ \\
$\gamma$ & Mass of the rotor & $98165 \mathrm{~kg}$ \\
$k$ & Shaft stiffness & $3.456 \cdot 10^{8} \mathrm{~N} / \mathrm{m}$ \\
$\omega$ & Rotor rotation speed & $14.2 \mathrm{rad} / \mathrm{s}$ \\
$k_{m}$ & Magnetic stiffness & $1.4715 \cdot 10^{8} \mathrm{~N} / \mathrm{m}$ \\
$\mu_{0}$ & Permeability of air & $4 \pi \cdot 10^{-7} \mathrm{Vs} / \mathrm{Am}$ \\
& Number of poles & 44 \\
\hline \hline
\end{tabular}

TABLE II: $\psi$ for $m, n \in[1,10]$. Locations where the UMP is zero are indicated with $\mathbf{X}$.

\begin{tabular}{|c|c|c|c|c|c|c|c|c|c|c|}
\hline $\mathbf{m} \backslash \mathbf{n}$ & $\mathbf{1}$ & $\mathbf{2}$ & $\mathbf{3}$ & $\mathbf{4}$ & $\mathbf{5}$ & $\mathbf{6}$ & $\mathbf{7}$ & $\mathbf{8}$ & $\mathbf{9}$ & $\mathbf{1 0}$ \\
\hline $\mathbf{1}$ & +1 & +2 & +3 & +4 & +5 & +6 & +7 & +8 & +9 & +10 \\
\hline $\mathbf{2}$ & +1 & $\mathbf{X}$ & +3 & $\mathbf{X}$ & +5 & $\mathbf{X}$ & +7 & $\mathbf{X}$ & +9 & $\mathbf{X}$ \\
\hline $\mathbf{3}$ & +1 & -2 & $\mathbf{X}$ & +4 & -5 & $\mathbf{X}$ & +7 & -8 & $\mathbf{X}$ & +10 \\
\hline $\mathbf{4}$ & +1 & $\mathbf{X}$ & -3 & $\mathbf{X}$ & +5 & $\mathbf{X}$ & -7 & $\mathbf{X}$ & +9 & $\mathbf{X}$ \\
\hline $\mathbf{5}$ & +1 & -4 & +6 & -4 & $\mathbf{X}$ & +6 & -14 & +16 & -9 & $\mathbf{X}$ \\
\hline $\mathbf{6}$ & +1 & $\mathbf{X}$ & $\mathbf{X}$ & $\mathbf{X}$ & -5 & $\mathbf{X}$ & +7 & $\mathbf{X}$ & $\mathbf{X}$ & $\mathbf{X}$ \\
\hline $\mathbf{7}$ & +1 & -6 & -6 & +8 & +15 & -6 & $\mathbf{X}$ & +8 & -27 & -20 \\
\hline $\mathbf{8}$ & +1 & $\mathbf{X}$ & +9 & $\mathbf{X}$ & -15 & $\mathbf{X}$ & -7 & $\mathbf{X}$ & +9 & $\mathbf{X}$ \\
\hline $\mathbf{9}$ & +1 & -8 & $\mathbf{X}$ & -8 & +10 & $\mathbf{X}$ & +28 & -8 & $\mathbf{X}$ & +10 \\
\hline $\mathbf{1 0}$ & +1 & $\mathbf{X}$ & -9 & $\mathbf{X}$ & $\mathbf{X}$ & $\mathbf{X}$ & +21 & $\mathbf{X}$ & -9 & $\mathbf{X}$ \\
\hline
\end{tabular}




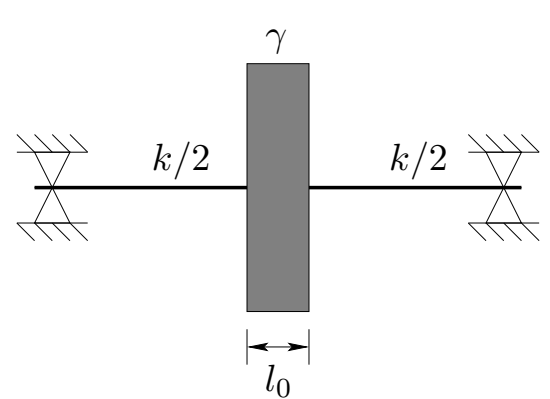

(a) The Jeffcott rotor.

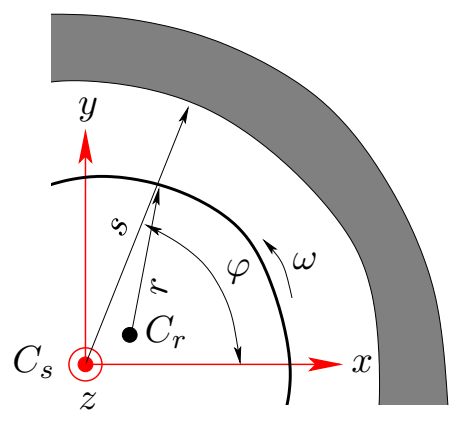

(b) The cross-section of the

FIG. 1: The generator model.

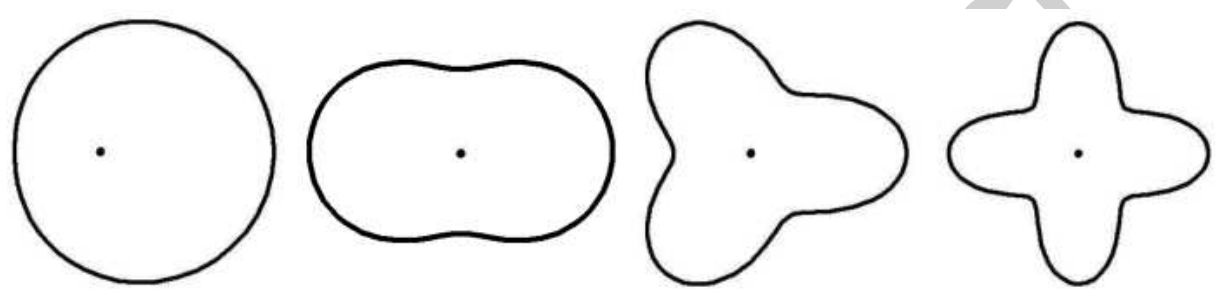

FIG. 2: The shape of the rotor for $\delta_{n}^{r}=r_{0} / 3, n=1,2,3,4$.

FIG. 3: The volume element $d V$. 


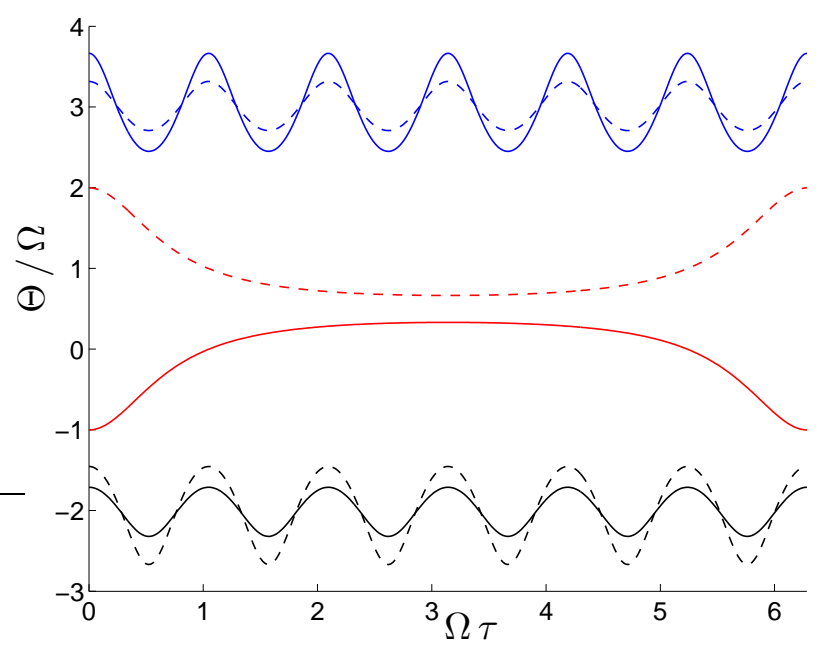

FIG. 4: The angular frequency ratio $\Theta / \Omega$ for $\Delta_{m}^{S}=0.05, \Delta_{n}^{R}=0.1$ (dashed) and $\Delta_{m}^{S}=$ $0.1, \Delta_{n}^{R}=0.05$ (solid). Three $m n$-combinations are considered, $[m, n]=[2,3]$ (positive), $[3,2]$ (negative) and $[1,1]$ (alternating).

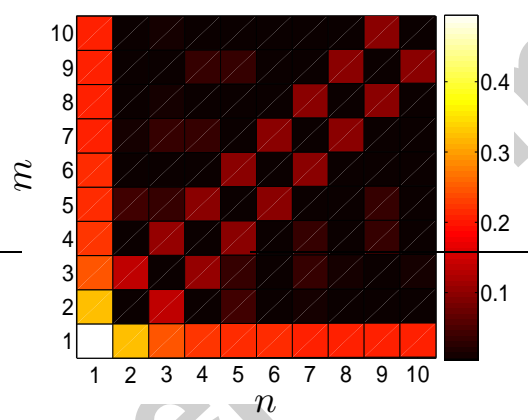

(a) The maximum amplitude for $m, n \in[1,10], \Delta_{m}^{S}=\Delta_{n}^{R}=0.3$.

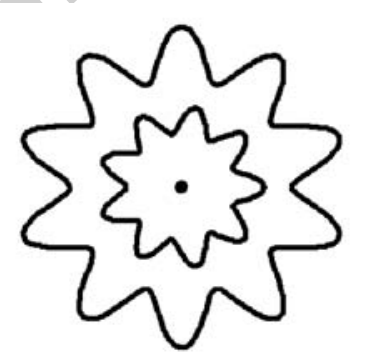

(b) The generator geometry for $\Delta_{10}^{S}>0$,

$$
\Delta_{9}^{R}>0 .
$$

FIG. 5: The amplitude of the UMP. 


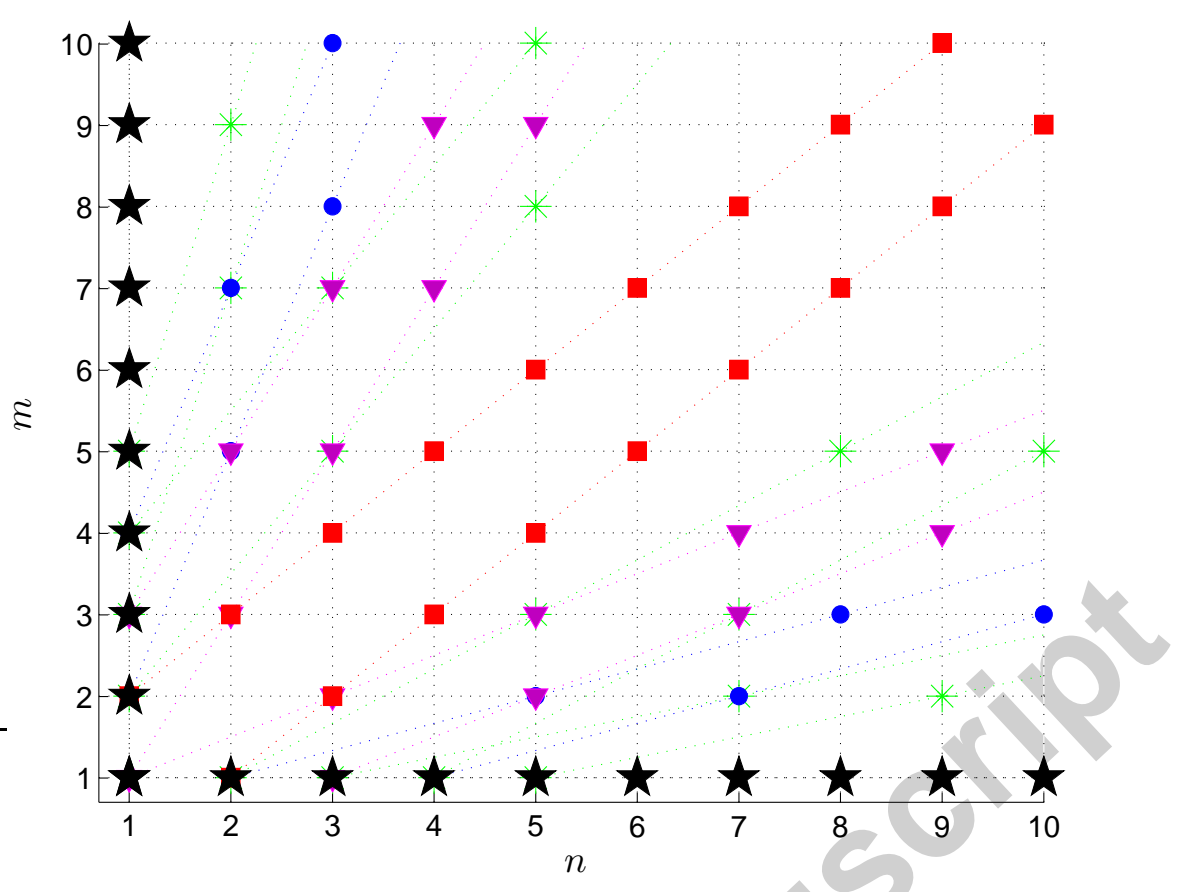

FIG. 6: Amplitude of the UMP in the $m n$-plane for $m, n \in[0,10]$. The points with nonzero terms from $F_{X}^{q}$ and $F_{Y}^{q}$ is marked according to; $q=1(\boldsymbol{\star}), q=2(\boldsymbol{\bullet}), q=3(\boldsymbol{\nabla}), q=4(\bullet)$ and $q=5(*)$. 


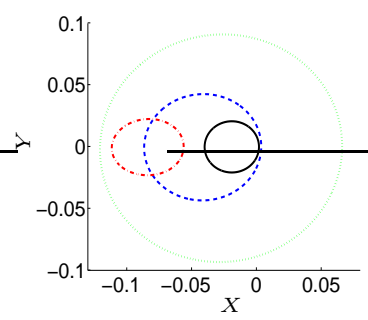

(a) $(1,1), 1$.

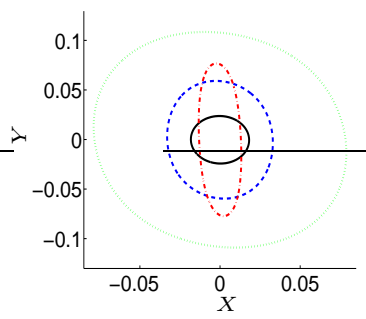

(d) $(2,1), 1$.

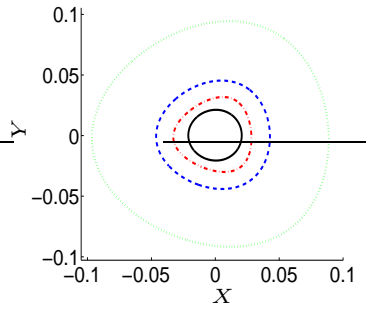

(g) $(3,1), 1$.

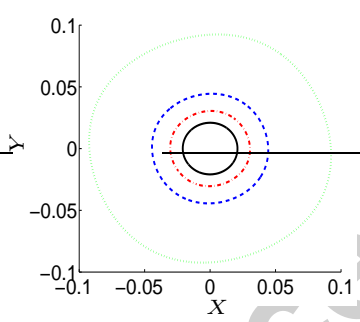

(j) $(4,1), 1$.

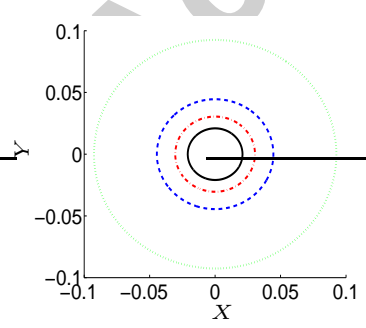

(m) $(5,1), 1$.

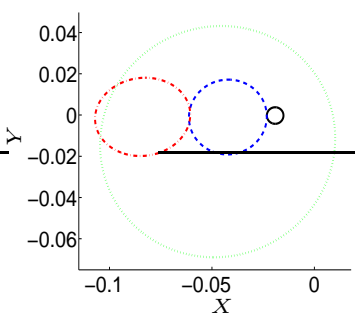

(b) $(1,2), 2$.

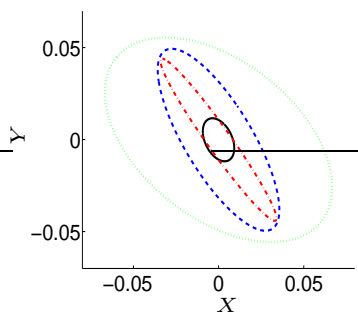

(e) $(2,3), 3$.

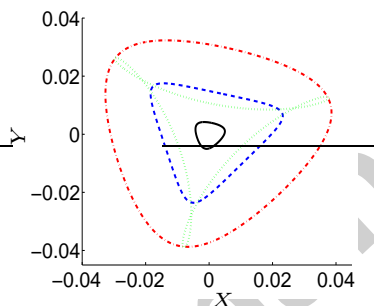

(h) $(3,2),-2$.

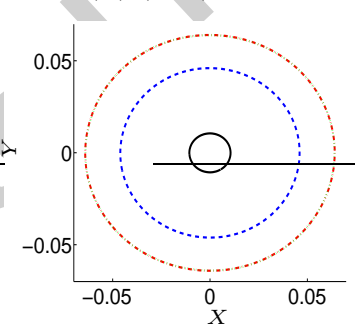

(k) $(4,3),-3$.

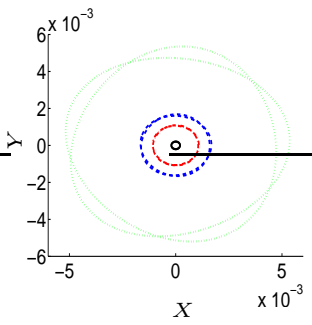

(n) $(5,2),-4$.

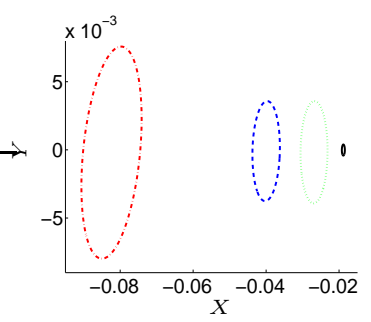

(c) $(1,3), 3$.

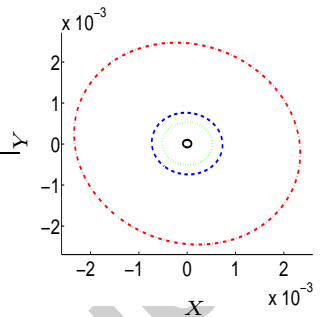

(f) $(2,5), 5$.

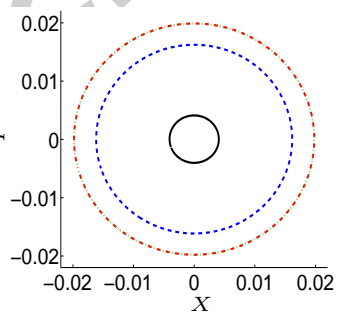

(i) $(3,4), 4$.

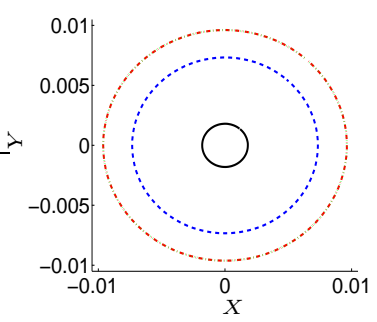

(1) $(4,5), 5$.

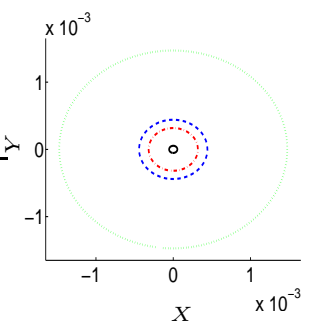

(о) $(5,3), 6$.

FIG. 7: Periodic attractors for the shape perturbations $\left(\Delta_{n}^{R}\left[\Delta_{1}^{R}\right], \Delta_{m}^{S}\left[\Delta_{1}^{S}\right]\right)=$ $(0.05[0.025], 0.05[0.025])$ (solid), (0.1[0.05], 0.1[0.05]) (dashed), (0.05[0.025], 0.25[0.1]) (dash-dot) and $(0.25[0.1], 0.05[0.025])$ (dotted). In each subfigure, the $m n$-combination and the corresponding whirling is given by the notation $(m, n), \psi$. 


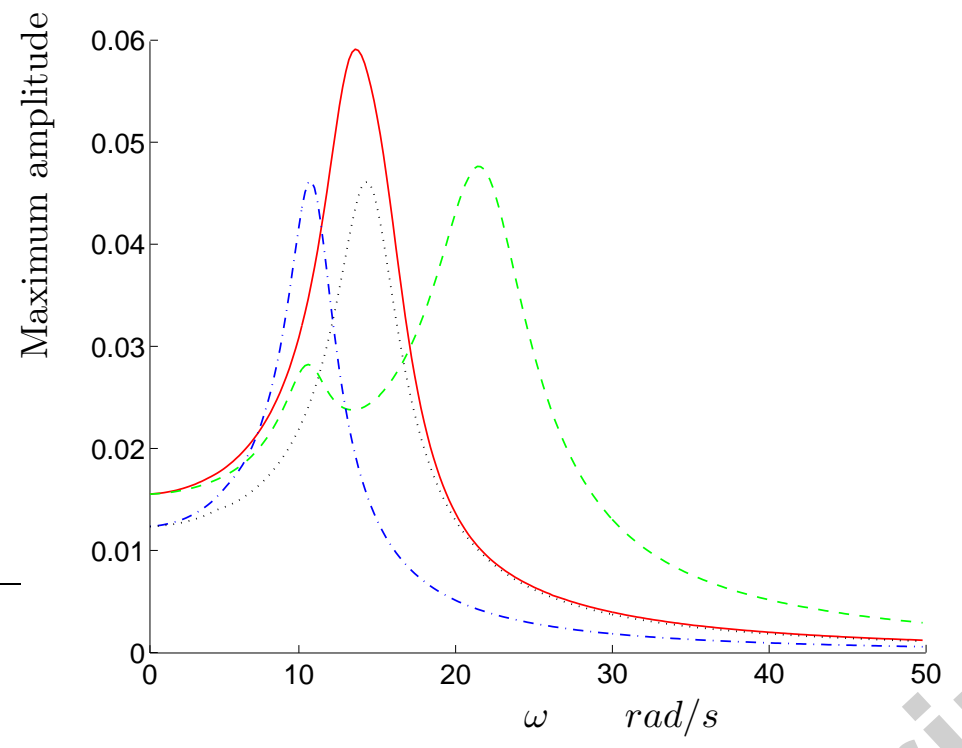

FIG. 8: Maximum amplitude as a function of $\omega$ for $[m, n]=[2,3]$ (solid), [3,2] (dashed) $[3,4]$ (dash-dot), [4,3] (dotted) with $\Delta_{n}^{R}=\Delta_{m}^{S}=0.1$. 